\title{
Fixed-dose combination therapy for the prevention of cardiovascular disease
}

\author{
Angharad N de Cates ${ }^{1}$, Matthew RB Farr ${ }^{1}$, Karen Rees ${ }^{1}$, Juan P Casas ${ }^{2}$, and Mark Huffman ${ }^{3}$ \\ ${ }^{1}$ Division of Health Sciences, Warwick Medical School, University of Warwick, Coventry, UK. \\ ${ }^{2}$ Department of Non-communicable Disease Epidemiology, London School of Hygiene and \\ Tropical Medicine; Department of Epidemiology and Public Health, University College London, \\ London, UK. \\ ${ }^{3}$ Departments of Preventative Medicine and Medicine (Cardiology), Northwestern University \\ Feinberg School of Medicine, Chicago, USA
}

\begin{abstract}
This is the protocol for a review and there is no abstract. The objectives are as follows:

To determine the effectiveness of fixed-dose combination therapy on optimising CVD risk factors and reducing CVD fatal and non-fatal events for both primary and secondary prevention of CVD. Details of CVD events and risk factors included are listed in the methods. We will also determine any adverse events associated with taking fixed-dose combination therapy. This will include studies conducted in both developed and developing regions of the world.
\end{abstract}

\section{BACKGROUND}

\section{Description of the condition}

Cardiovascular disease (CVD) is a principal cause of death worldwide (WHO 2005). In 2008, it was directly responsible for more than 17 million deaths globally, over $80 \%$ of which occurred in low and middle-income countries (WHO 2010 (a)). Furthermore, the situation is not expected to improve, with global CVD mortality estimated to increase by six million over the next 20 years (WHO 2010 (a)). Ischaemic heart disease and cerebrovascular diseases - the major CVDs - are also major causes of disability resulting in 35 million disability-adjusted life years (DALYs) lost in 2004 (WHO 2004). Therefore, preventing deaths and disease due to CVD is a priority for global public health.

Copyright $(92012$ The Cochrane Collaboration. Published by John Wiley \& Sons, Ltd.

Contact address: Juan P Casas, Department of Non-communicable Disease Epidemiology, London School of Hygiene and Tropical Medicine; Department of Epidemiology and Public Health, University College London, Keppel Street, London, WC1E 7HT, UK. juan.pablo-casas@1shtm.ac.uk.

Editorial group: Cochrane Heart Group.

Publication status and date: New, published in Issue 5, 2012.

CONTRIBUTIONS OF AUTHORS All authors contributed to the development of the protocol.

DECLARATIONS OF INTEREST None known. 
CVD is a multi-factorial disease. Risk factors can be divided into non-modifiable (including age and sex) and potentially modifiable. Worldwide case-control data demonstrate that in over $90 \%$ of cases an individual's first cardiovascular event relates to one or more of ten specific modifiable factors, which include hypertension and abnormal lipid profile (O'Donnell 2010; Yusuf 2004). Although the same risk factors are present in almost all populations, distribution has been shown to vary across regional and sociological lines (Steyn 2005). Hypertension is most prevalent in low and middle-income countries, whereas dyslipidaemia is most prevalent in high-income countries (WHO 2010 (a)). In 2008, approximately $40 \%$ of UK adults as opposed to $45 \%$ of South African adults older than 25 years had controlled or uncontrolled hypertension. In contrast, in the same year, prevalence of hyper-cholesterolaemia (total cholesterol greater than $5 \mathrm{mmol} / \mathrm{L}$ ) in the UK was $60 \%$ compared to 35\% in South Africa (WHO 2008). Almost 20\% of adults older than 20 years have both hypertension and dyslipidaemia in the US (Wong 2006), and fewer than $10 \%$ of US adults have no major CVD risk factors (Ford 2009). Therefore, modifiable risk factors are identifiable, prevalent in most populations, and often co-exist.

Optimising modifiable risk factors in an individual reduces overall cardiovascular risk and thereby reduces CVD mortality and morbidity (Cowie 2005). Individuals with both hypertension and dyslipidaemia have a greater risk of CVD than those with either hypertension or dyslipidaemia alone (Neaton 1992; Thomas 2002), highlighting the importance of considering overall CVD risk as opposed to individual risk factors (Graham 2007). Therefore, adopting a multi-factorial approach to CVD risk management, where multiple risk factors are modified simultaneously, is more effective at reducing CVD events than focusing on single risk factors in isolation (Gaede 2003).

Current national and international approaches to CVD prevention incorporate both primary and secondary prevention (Graham 2007; NICE 2010). Primary prevention aims to prevent CVD events in those who have no clinical evidence of CVD. To achieve this, and using defined validated risk scores, guidelines recommend intervening when the 10 year risk for any CVD event is estimated to be at $20 \%$, or when a fatal CVD event is estimated to be at 5\% (Graham 2007; NICE 2008; NICE 2010). Modifying risk factors in this population is known to reduce CVD risk and mortality by using antihypertensives to lower blood pressure (Collins 1990) and statins to improve lipid profile (Taylor 2011). Secondary prevention involves blood pressure control, cholesterol lowering and use of antiplatelet drugs to prevent further CVD events in those with pre-existing clinical evidence of CVD. This approach is also known to be effective (ATT-Collaboration 2002; Baigent 2005; Rashid 2003).

As the same CVD risk factors operate throughout the global population (Yusuf 2004), worldwide CVD prevention strategies need to be multi-factorial. However, implementing conventional multi-factorial CVD prevention approaches across the global population, that is, targeting high risk individuals with various medications and regular monitoring to optimise multiple CVD risk factors, highlights three major issues:

(i) Epidemiological evidence suggests reducing blood pressure, cholesterol and smoking each by $10 \%$ across the UK adult population would save over 9000 lives in 10 years (Cooney 2009), significantly more than if a similar approach were targeted only at high 
risk individuals (Cooney 2009). Therefore, a small reduction in cardiovascular risk across a large number of people may be more effective at preventing CVD events in the population than treating a small number of people at high risk (NICE 2010).

(ii) Lipid-lowering therapy in particular has been demonstrated to reduce CVD events when pre-treatment lipid levels are normal (Colhoun 2004; HPSCG 2002; O'Keefe 2004; Sever 2003). This suggests that some with a potential to benefit from therapy may be excluded under existing CVD prevention programmes.

(iii) Implementing the conventional CVD prevention strategy to the whole population would be practically challenging, due to the financial and time costs of monitoring, including the need for regular blood samples, and the administration issues of mass prescriptions of multiple tablets.

Therefore, because of the global burden of CVD and the issues with implementing conventional strategies worldwide, an alternative and complementary population-wide strategy is required.

\section{Description of the intervention}

In 2001, a meeting of experts co-ordinated by the World Health Organisation (WHO) and Wellcome Trust determined that the use of a combination (fixed-dose) pill may be beneficial for secondary prevention of CVD (WHO 2002). A year later, Yusuf proposed that a combination of four specific drugs (beta blocker, aspirin, statin and ACE-inhibitor) could reduce CVD events by 75\% in those already suffering from CVD (Yusuf 2002). In 2003, Wald and Law first proposed their Polypill (a combination of folic acid, aspirin, three lowdose antihypertensives and a low-dose statin) with the aim to reduce several cardiovascular risk factors simultaneously (Wald 2003). They estimated that administration to all with CVD (i.e. secondary prevention) as well as to all those aged 55 years and over without CVD (i.e. modified primary prevention) would safely reduce myocardial infarction by $88 \%$ and stroke by $80 \%$ (Wald 2003). Recent evidence has indicated that the effects of fixed-dose combination treatment may be less than initially proposed, but that this strategy may improve blood pressure and lipid profile to near expected levels (TIPS 2009), and that in fact CVD event reduction of up to $50 \%$ by this route may be feasible (PILL-collaborative 2011). The controversial aspect of Wald and Law's approach was the suggestion that the fixed-dose combination therapy could be administered at a population level without expensive screening of blood cholesterol or blood pressure (Wald 2011). Instead a threshold age, such as above 55, would be used as the entrance criterion (Lonn 2010; Wald 2003).

Recent evidence has supported aspirin for secondary prevention but has questioned its use for primary prevention: the potential cost of serious bleeding may outweigh any CVD risk reduction benefit (Baigent 2009). Also, doubt exists regarding folic acid, since recent large randomised trials indicated no CVD benefit (Armitage 2010; Holmes 2011). On the other hand, statins and antihypertensives as single treatments are known to be relatively safe and beneficial individually in terms of reducing CVD risk and thereby cardiovascular events for secondary prevention and high-risk primary prevention (ALLHAT-investigators 2002; Colhoun 2004; HPSCG 2002; Julius 2004; Kearney 2008; LaRosa 2005; Ostergren 2008; Papademetriou 2003; Sever 2003;Turnbull 2003). Therefore, although uncertainty exists 
regarding possible components, the consensus is that the ideal fixed-dose combination therapy for primary and secondary CVD prevention should include at least one antihypertensive and one statin.

There is some recent evidence regarding efficacy and safety of antihypertensives and statins when administered concomitantly (Messerli 2006; Preston 2007), and of multiple antihypertensives when administered as a single tablet (Gupta 2010). However, there are concerns that clinicians may be wary of combination therapy, due to the potential restrictions on individualised management (Viera 2011); that is, the ability to amend standard therapy because of medical history or adverse events, such as avoiding a betablocker in an asthmatic or changing from an ACE-inhibitor due to cough, and to titrate each drug prescribed according to clinical response (Lonn 2010).

\section{How the intervention might work}

According to the Grading of Recommendations Assessment, Development and Evaluation (GRADE) Working Group, how strongly an intervention can be recommended to patients, clinicians and policymakers is determined by four factors: the balance between beneficial and adverse effects, the quality of evidence, the variability in preferences of those involved, and costs (Guyatt 2008). Pharmacological therapy has been postulated by some as the population-level strategy with the most promise to reduce global CVD risk (Beaglehole 2011; Lonn 2010). This is because there is limited evidence of effectiveness regarding behavioural and lifestyle interventions (Ebrahim 2011) and although modifying national health policy has been successful in developed countries such as Scandinavia (Vartiainen 2010), it is expensive and can be politically challenging, especially in developing countries (Lonn 2010). However, the fixed-dose combination therapy is likely to have a greater potential for adverse effects than behavioural or lifestyle changes, and some argue that a purely biological approach is too narrow to allow the social, economic and behavioural complexities of CVD prevention to be appreciated and confronted (Franco 2004).

Both statins and antihypertensives delivered separately or together, reduce CVD risk and therefore CVD events in high risk populations and in those with pre-existing CVD. However, despite known effectiveness, not all those with a potential to benefit are prescribed therapies (Kotseva 2009; Mendis 2005), and many are non-concordant (Osterberg 2005). In some countries, therapies cannot be afforded (Mendis 2005). Recent global epidemiological data from the PURE investigators indicates that overall use of secondary prevention medication was less than $30 \%$, and that levels of use are particularly poor in low to middle-income countries and in rural regions (Yusuf 2011). The likely result is inadequate prevention of further CVD events.

There is a potential for these issues to be resolved by fixed-dose combination therapy. Prescribing and administering a single tablet to modify multiple risk factors simultaneously without the need for intensive monitoring would be practical and desirable. Implementing fixed-dose combination therapy for secondary prevention of CVD is a much smaller extension from current practice, and so is less controversial. Most patients would already be prescribed the individual components, and defining those with a potential to benefit is far easier due to the presence of clinical symptoms. However, two possibilities have been 
suggested for the more contentious global fixed-dose combination therapy primary prevention strategy: administer to the whole population above a threshold age as suggested by the original Polypill@ (Wald 2003;Wald 2011), or administer to individuals identified as high risk either with or without blood sample screening and monitoring (Beaglehole 2011). Modified risk assessment charts have been produced for which blood samples are not necessary for risk stratification (Gaziano 2008; WHO 2010 (b)). Using these charts to avoid expensive screening and monitoring, estimates suggest that a fixed-dose combination therapy implemented across 23 low and middle-income countries solely to those identified as high risk would cost approximately US $\$ 1$ per person per annum (Lim 2007), an amount likely to be affordable.

However, fixed-dose combination therapy still has many unknowns: (i) the best constituents: whether two or three or four or five drugs are required and (ii) evidence of safety, effectiveness and cost-effectiveness: whether increasing the number of constituents will produce a favourable risk: benefit profile and be worth the increased cost. In particular, the evidence is sparse concerning fixed-dose combination therapy, both benefits and adverse effects, for primary prevention in those with low CVD risk. Further knowledge concerning this last point is essential if fixed-dose combination therapy is to be considered for administration to the entire population above a threshold age. Finally, several authors have questioned whether fixed-dose combination strategy may have unforeseen negative impacts on other aspects of CVD risk reduction e.g. individuals neglecting to exercise because of a sense of CVD security with fixed-dose combination therapy (Lonn 2010). As yet there is no long-term follow-up outcome and safety data, which is of particular concern considering that the original Polypill concept was designed with long-term use of fixed-dose combination therapy in mind.

\section{Why it is important to do this review}

Various fixed-dose combination pills are now in manufacture and there is evidence that physicians are aware of this option and are potentially willing to prescribe it (Viera 2011). There is an emerging literature of randomised controlled trials comparing fixed-dose combination therapy with placebo or standard practice in both primary and secondary prevention of CVD, as well as assessing safety and tolerability. However, to date, the evidence has not been synthesised in a systematic review.

\section{OBJECTIVES}

To determine the effectiveness of fixed-dose combination therapy on optimising CVD risk factors and reducing CVD fatal and non-fatal events for both primary and secondary prevention of CVD. Details of CVD events and risk factors included are listed in the methods. We will also determine any adverse events associated with taking fixed-dose combination therapy. This will include studies conducted in both developed and developing regions of the world. 


\section{METHODS}

\section{Criteria for considering studies for this review}

Types of studies-Randomised controlled trials.

Types of participants-Adults older than 18 years, with no restriction regarding presence of CVD.

Types of interventions-A fixed-dose combination therapy: a combination of several active components into a single pill with the aim to optimise CVD risk and reduce CVD fatal and non-fatal events. At least one statin and one antihypertensive agent should be included. Different combinations and doses will be examined in stratified analyses if there are sufficient trials.

Trials will be considered where the comparison group is standard practice or placebo. Standard practice will vary between trials depending on location of study. Proportion of participants undergoing existing treatment aimed at reducing CVD events or risk, and specifics of treatment, will be noted.

\section{Types of outcome measures}

Primary outcomes: Clinical outcomes such as mortality (cardiovascular and all-cause); non-fatal CVD endpoints such as myocardial infarction, coronary artery bypass grafting (CABG), percutaneous transluminal coronary angioplasty (PTCA), those with angina or angiographically defined CHD, stroke, transient ischaemic attack (TIA), carotid endarterectomy, peripheral arterial disease (PAD).

Secondary outcomes: Blood pressure (systolic and diastolic), lipid levels (total cholesterol, high density lipoprotein (HDL), low density lipoprotein (LDL) and triglycerides).

Health related quality of life, measured according to any well-validated and adjusted scale concerning quality of life.

Adverse effects, including overall rates of discontinuation, proportion of participants experiencing specific symptoms / results and rates of discontinuation by specific symptoms / results (including but not limited to: electrolyte disturbances, myalgia, elevated liver enzymes). We will also consider adherence to therapy and any changes in medication throughout the trials.

Costs of fixed-dose combination therapy.

\section{Search methods for identification of studies}

Electronic searches-The following electronic databases will be searched: The Cochrane Library (including the Cochrane Central Register of Controlled Trials (CENTRAL), DARE, HTA and NHS EDD), MEDLINE, EMBASE and Web of Science. Medical subject headings (MeSH) or equivalent and text word terms will be used. Searches will be designed in accordance with Cochrane Heart Group methods and guidance. There 
will be no language restrictions. The searches have been limited to records published since 2000. The fixed-dose combination therapy was conceptualised in 2001, and so relevant trials will only appear after this date.

Searches will be tailored to individual databases. The search strategy for MEDLINE is shown in Appendix 1.

Searching other resources-We will search the metaRegister of controlled trials (mRCT) (www.controlled-trials.com/mrct), clinicaltrials.gov (www.clinicaltrials.gov) and the WHO International Clinical Trials Registry Platform (ICTRP) (http://apps.who.int/ trialsearch/) for ongoing trials. In addition, reference lists of reviews and retrieved articles will be checked for additional studies and citation searches will be performed on key articles. Experts in the field will be contacted for unpublished and ongoing trials. Authors will be contacted where necessary for additional information.

\section{Data collection and analysis}

Selection of studies-From the searches, the title and abstract of each paper will be reviewed by two reviewers and potentially relevant references retrieved. Following this initial screening, the full text reports of potentially relevant studies will be obtained, and two reviewers (AdC and MF) will independently select studies to be included in the review using predetermined inclusion criteria. In all cases disagreements about any study inclusions will be resolved by consensus and a third reviewer will be consulted if disagreement persists.

Data extraction and management-Data will be extracted independently by two reviewers (AdC and MF) using a proforma and chief investigators will be contacted to provide additional relevant information if necessary. Details of the study design, participant characteristics, study setting, intervention and comparator, and outcome data including details of outcome assessment, adverse effects and methodological quality (randomisation, blinding, attrition) will be extracted from each of the included studies. Disagreements about extracted data will be resolved by consensus and a third reviewer will be consulted if disagreement persists.

Assessment of risk of bias in included studies-Risk of bias will be assessed according to the Cochrane Risk of Bias Assessment tool, including examining the quality of the random sequence generation and allocation concealment, description of drop-outs and withdrawals (including analysis by intention-to-treat), blinding (participants, personnel, and outcome assessment) and selective outcome reporting (Higgins 2011). The risk of bias in included studies will be assessed independently by two reviewers.

Measures of treatment effect-Data will be processed in accordance with the Cochrane Handbook for Systematic Reviews of Interventions (Higgins 2011). Dichotomous outcomes will be expressed as odds ratios or relative risks, and 95\% confidence intervals (CI) will be calculated for each study. For continuous variables net changes will be compared (i.e. intervention group minus control group differences) and a weighted mean difference (WMD) or standardised mean difference (SMD) and 95\% CIs will be calculated for each study. 
Assessment of heterogeneity-For each outcome tests of heterogeneity will be carried out (using the $\mathrm{chi}^{2}$ test of heterogeneity and $\mathrm{I}^{2}$ statistic). In the situation of no heterogeneity a fixed-effect meta-analysis will be performed. If substantial heterogeneity is detected the reviewers will look for possible explanations for this (e.g. participants and intervention). If the heterogeneity cannot be explained, the reviewers will consider the following options: provide a narrative overview and not aggregate the studies at all, or use a random-effects model with appropriate cautious interpretation.

Subgroup analysis and investigation of heterogeneity-If there are sufficient studies we will conduct the following prespecified subgroup analyses:

1. Age.

2. Sex.

3. Focus of study is primary prevention (populations where $10 \%$ or less have preexisting CVD) versus secondary prevention (population where majority have preexisting CVD).

4. High dose/potency of antihypertensive and statin used versus lower dose. These are relative terms based on what is reported in the included trials and do not relate to specific doses.

5. Two versus three or more fixed-dose drugs used.

Usual care will be as defined by the study. If applicable, results within usual care group will be stratified and analysed separately.

Sensitivity analysis-Sensitivity analyses will be carried out excluding studies of low methodological quality. Funnel plots and tests of asymmetry (Egger 1997) will be undertaken to assess possible publication bias.

\section{Acknowledgments}

\section{SOURCES OF SUPPORT}

Internal sources

- Warwick Medical School, University of Warwick, UK

- Department of Non-Communicable Disease Epidemiology, London School of Hygiene and Tropical Medicine, UK.

External sources

- $\quad$ NIHR Cochrane Programme Grant, UK.

\section{Appendix 1. MEDLINE search strategy MEDLINE OVID}

The Cochrane sensitivity-maximising RCT filter has been used (Lefebvre 2011)

1. exp Cardiovascular Diseases/

2. cardio*.tw. 
3. cardia*.tw.

4. heart*.tw.

5. coronary*.tw.

6. angina*.tw.

7. ventric*.tw.

8. myocard*.tw.

9. pericard*.tw.

10. isch?em*.tw.

11. emboli*.tw.

12. arrhythmi*.tw.

13. thrombo*.tw.

14. atrial fibrillat*.tw.

15. tachycardi*.tw.

16. endocardi*.tw.

17. (sick adj sinus).tw.

18. exp Stroke/

19. (stroke or stokes).tw.

20. cerebrovasc*.tw.

21. cerebral vascular.tw.

22. apoplexy.tw.

23. (brain adj2 accident*).tw.

24. ((brain* or cerebral or lacunar) adj2 infarct*).tw.

25. exp Hypertension/

26. hypertensi*.tw.

27. peripheral arter* disease*.tw.

28. ((high or increased or elevated) adj2 blood pressure).tw.

29. exp Hyperlipidemias/

30. hyperlipid*.tw.

31. hyperlip?emia*.tw.

32. hypercholesterol*.tw.

33. hypercholester?emia*.tw.

34. hyperlipoprotein?emia*.tw. 
35. hypertriglycerid?emia*.tw.

36. or/1-35

37. Drug Combinations/

38. polypill*.tw.

39. (drug adj2 combin*).tw.

40. ((multi* or several) adj2 (ingredient* or component*)).tw.

41. policap.tw.

42. quintapill.tw.

43. (single adj2 pill* adj 2 comb*).tw.

44. single-pill.tw.

45. Red Heart pill*.tw.

46. or $/ 37-45$

47. 36 and 46

48. randomized controlled trial.pt.

49. controlled clinical trial.pt.

50. randomized.ab.

51. placebo.ab

52. drug therapy.fs.

53. randomly.ab.

54. trial.ab.

55. groups.ab.

56. 48 or 49 or 50 or 51 or 52 or 53 or 54 or 55

57. exp animals/ not humans.sh.

58. 56 not 57

59. 47 and 58

60. limit 59 to $\mathrm{yr}=" 2000$-Current"

\section{HISTORY}

Protocol first published: Issue 5, 2012

\section{Additional references}

ALLHAT-investigators 2002 . ALLHAT-investigators. Major outcomes in high-risk hypertensive patients randomized to angiotensin-converting enzyme inhibitor or calcium channel blocker vs 
diuretic: The Antihypertensive and Lipid-Lowering Treatment to Prevent Heart Attack Trial (ALLHAT). JAMA. 2002; 288(23):2981-97. [PubMed: 12479763]

Armitage 2010 . Armitage JM, Bowman L, Clarke RJ, Wallendszus K, Bulbulia R, Rahimi K, et al. Effects of homocysteine-lowering with folic acid plus vitamin B12 vs placebo on mortality and major morbidity in myocardial infarction survivors: a randomized trial. JAMA. 2010; 303(24): 2486-94. [PubMed: 20571015]

ATT-Collaboration 2002 . ATT-Collaboration. Collaborative meta-analysis of randomised trials of antiplatelet therapy for prevention of death, myocardial infarction, and stroke in high risk patients. BMJ. 2002; 324(7329):71-86. [PubMed: 11786451]

Baigent 2005 . Baigent C, Keech A, Kearney PM, Blackwell L, Buck G, Pollicino C, et al. Efficacy and safety of cholesterol-lowering treatment: prospective meta-analysis of data from 90,056 participants in 14 randomised trials of statins. Lancet. 2005; 366(9493):1267-78. [PubMed: 16214597]

Baigent 2009 . Baigent C, Blackwell L, Collins R, Emberson J, Godwin J, Peto R, et al. Aspirin in the primary and secondary prevention of vascular disease: collaborative meta-analysis of individual participant data from randomised trials. Lancet. 2009; 373(9678):1849-60. [PubMed: 19482214]

Beaglehole 2011 . Beaglehole R, Bonita R, Horton R, Adams C, Alleyne G, Asaria P, et al. Priority actions for the non-communicable disease crisis. Lancet. 2011; 377(9775):1438-47. [PubMed: 21474174]

Colhoun 2004 . Colhoun HM, Betteridge DJ, Durrington PN, Hitman GA, Neil HA, Livingstone SJ, et al. Primary prevention of cardiovascular disease with atorvastatin in type 2 diabetes in the Collaborative Atorvastatin Diabetes Study (CARDS): multicentre randomised placebo-controlled trial. Lancet. 2004; 364(9435):685-96. [PubMed: 15325833]

Collins 1990 . Collins R, Peto R, MacMahon S, Hebert P, Fiebach NH, Eberlein KA, et al. Blood pressure, stroke, and coronary heart disease. Part 2, Short-term reductions in blood pressure: overview of randomised drug trials in their epidemiological context. Lancet. 1990; 335(8693): 827-38. [PubMed: 1969567]

Cooney 2009 . Cooney MT, Dudina A, Whincup P, Capewell S, Menotti A, Jousilahti P, et al. Reevaluating the Rose approach: comparative benefits of the population and high-risk preventive strategies. Eur J Cardiovasc Prev Rehabil. 2009; 16(5):541-9. [PubMed: 19773660]

Cowie 2005 . Cowie MR. Simultaneous treatment of hypertension and dyslipidaemia may help to reduce overall cardiovascular risk: focus on amlodipine/atorvastatin single-pill therapy. Int J Clin Pract. 2005; 59(7):839-46. [PubMed: 15963213]

Ebrahim 2011 . Ebrahim S, Taylor F, Ward K, Beswick A, Burke M, Davey Smith G. Multiple risk factor interventions for primary prevention of coronary heart disease. Cochrane Database of Systematic Reviews. 2011; (Issue 1) [DOI: 10.1002/14651858.CD001561.pub3].

Egger 1997 . Egger M, Davey Smith G, Schneider M, Minder C. Bias in meta-analysis detected by a simple, graphical test. BMJ. 1997; 315(7109):629-34. [PubMed: 9310563]

Ford 2009 . Ford ES, Li C, Zhao G, Pearson WS, Capewell S. Trends in the prevalence of low risk factor burden for cardiovascular disease among United States adults. Circulation. 2009; 120(13): 1181-8. [PubMed: 19752328]

Franco 2004 . Franco OH, Bonneux L, de Laet C, Peeters A, Steyerberg EW, Mackenbach JP. The Polymeal: a more natural, safer, and probably tastier (than the Polypill) strategy to reduce cardiovascular disease by more than 75\%. BMJ. 2004; 329(7480):1447-50. [PubMed: 15604180]

Gaede 2003 . Gaede P, Vedel P, Larsen N, Jensen GV, Parving HH, Pedersen O. Multifactorial intervention and cardiovascular disease in patients with type 2 diabetes. N Engl J Med. 2003; 348(5):383-93. [PubMed: 12556541]

Gaziano 2008 . Gaziano TA, Young CR, Fitzmaurice G, Atwood S, Gaziano JM. Laboratory-based versus non-laboratory-based method for assessment of cardiovascular disease risk: the NHANES I Follow-up Study cohort. Lancet. 2008; 371(9616):923-31. [PubMed: 18342687]

Graham 2007 . Graham I, Atar D, Borch-Johnsen K, Boysen G, Burell G, Cifkova R, et al. European guidelines on cardiovascular disease prevention in clinical practice: executive summary. Fourth Joint Task Force of the European Society of Cardiology and other societies on cardiovascular 
disease prevention in clinical practice (constituted by representatives of nine societies and by invited experts). Eur J Cardiovasc Prev Rehabil. 2007; 14(Suppl 2):E1-40. [PubMed: 17726406]

Gupta 2010 . Gupta AK, Arshad S, Poulter NR. Compliance, safety, and effectiveness of fixed-dose combinations of antihypertensive agents: a meta-analysis. Hypertension. 2010; 55(2):399-407. [PubMed: 20026768]

Guyatt 2008 . Guyatt GH, Oxman AD, Kunz R, Falck-Ytter Y, Vist GE, Liberati A, et al. Going from evidence to recommendations. BMJ. 2008; 336(7652):1049-51. [PubMed: 18467413]

Higgins 2011 . Higgins, JPT.; Green, S., editors. Cochrane Handbook for Systematic Reviews of interventions. Version 5.1.0. The Cochrane Collaboration; 2011. Available from www.cochranehandbook.org [updated March 2011]

Holmes 2011 . Holmes MV, Newcombe P, Hubacek JA, Sofat R, Ricketts SL, Cooper J, et al. Effect modification by population dietary folate on the association between MTHFR genotype, homocysteine, and stroke risk: a meta-analysis of genetic studies and randomised trials. Lancet. 2011; 378(9791):584-94. [PubMed: 21803414]

HPSCG 2002 . HPSCG. MRC/BHF Heart Protection Study of cholesterol lowering with simvastatin in 20,536 high-risk individuals: a randomised placebo-controlled trial. Lancet. 2002; 360(9326): 7-22. [PubMed: 12114036]

Julius 2004 . Julius S, Kjeldsen SE, Weber M, Brunner HR, Ekman S, Hansson L, et al. Outcomes in hypertensive patients at high cardiovascular risk treated with regimens based on valsartan or amlodipine: the VALUE randomised trial. Lancet. 2004; 363(9426):2022-31. [PubMed: 15207952]

Kearney 2008 . Kearney PM, Blackwell L, Collins R, Keech A, Simes J, Peto R, et al. Efficacy of cholesterol-lowering therapy in 18,686 people with diabetes in 14 randomised trials of statins: a meta-analysis. Lancet. 2008; 371(9607):117-25. [PubMed: 18191683]

Kotseva 2009 . Kotseva K, Wood D, De Backer G, De Bacquer D, Pyorala K, Keil U. EUROASPIRE III: a survey on the lifestyle, risk factors and use of cardioprotective drug therapies in coronary patients from 22 European countries. Eur J Cardiovasc Prev Rehabil. 2009; 16(2):121-37. [PubMed: 19287307]

LaRosa 2005 . LaRosa JC, Grundy SM, Waters DD, Shear C, Barter P, Fruchart JC, et al. Intensive lipid lowering with atorvastatin in patients with stable coronary disease. N Engl J Med. 2005; 352(14):1425-35. [PubMed: 15755765]

Lefebvre 2011 . Lefebvre, C.; Manheimer, E.; Glanville, J. Chapter 6: Searching for studies. In: Higgins, JPT.; Green, S., editors. Cochrane Handbook for Systematic Reviews of Interventions. Version 5.1.0. The Cochrane Collaboration; 2011. Available from www.cochrane-handbook.org [updated March 2011]

Lim 2007 . Lim SS, Gaziano TA, Gakidou E, Reddy KS, Farzadfar F, Lozano R, et al. Prevention of cardiovascular disease in high-risk individuals in low-income and middle-income countries: health effects and costs. Lancet. 2007; 370(9604):2054-62. [PubMed: 18063025]

Lonn 2010 . Lonn E, Bosch J, Teo KK, Pais P, Xavier D, Yusuf S. The polypill in the prevention of cardiovascular diseases: key concepts, current status, challenges, and future directions. Circulation. 2010; 122(20):2078-88. [PubMed: 21098469]

Mendis 2005 . Mendis S, Abegunde D, Yusuf S, Ebrahim S, Shaper G, Ghannem H, et al. WHO study on Prevention of REcurrences of Myocardial Infarction and StrokE (WHO-PREMISE). Bull World Health Organ. 2005; 83(11):820-9. [PubMed: 16302038]

Messerli 2006 . Messerli FH, Bakris GL, Ferrera D, Houston MC, Petrella RJ, Flack JM, et al. Efficacy and safety of coadministered amlodipine and atorvastatin in patients with hypertension and dyslipidemia: results of the AVALON trial. J Clin Hypertens (Greenwich). 2006; 8(8):57181. quiz 82-3. [PubMed: 16896273]

Neaton 1992 . Neaton JD, Wentworth D. Serum cholesterol, blood pressure, cigarette smoking, and death from coronary heart disease. Overall findings and differences by age for 316, 099 white men. Multiple Risk Factor Intervention Trial Research Group. Archives of Internal Medicine. 1992; 152(1):56-64. [PubMed: 1728930]

NICE 2008 . NICE. Lipid Modification. Cardiovascular risk assessment and the modification of blood lipids for the primary and secondary prevention of cardiovascular disease. 2008. http:// 
www.nice.org.uk/nicemedia/pdf/CG67NICEguideline.pdfhttp://www.nice.org.uk/nicemedia/pdf/ CG67NICEguideline.pdf

NICE 2010 . NICE. Prevention of cardiovascular disease at population level. 2010. http:// www.nice.org.uk/guidance/PH25http://www.nice.org.uk/guidance/PH25

O’Donnell 2010 . O’Donnell MJ, Xavier D, Liu L, Zhang H, Chin SL, Rao-Melacini P, et al. Risk factors for ischaemic and intracerebral haemorrhagic stroke in 22 counties (the INTERSTROKE study): a case-control study. Lancet. 2010; 376:112-23. [PubMed: 20561675]

O'Keefe 2004 . O'Keefe JH Jr, Cordain L, Harris WH, Moe RM, Vogel R. Optimal low-density lipoprotein is 50 to $70 \mathrm{mg} / \mathrm{dl}$ : lower is better and physiologically normal. J Am Coll Cardiol. 2004; 43(11):2142-6. [PubMed: 15172426]

Osterberg 2005 . Osterberg L, Blaschke T. Adherence to medication. N Engl J Med. 2005; 353(5): 487-97. [PubMed: 16079372]

Ostergren 2008 . Ostergren J, Poulter NR, Sever PS, Dahlof B, Wedel H, Beevers G, et al. The Anglo-Scandinavian Cardiac Outcomes Trial: blood pressure-lowering limb: effects in patients with type II diabetes. J Hypertens. 2008; 26(11):2103-11. [PubMed: 18854748]

Papademetriou 2003 . Papademetriou V, Piller LB, Ford CE, Gordon D, Hartney TJ, Geraci TS, et al. Characteristics and lipid distribution of a large, high-risk, hypertensive population: the lipidlowering component of the Antihypertensive and Lipid-Lowering Treatment to Prevent Heart Attack Trial (ALLHAT). J Clin Hypertens (Greenwich). 2003; 5(6):377-84. [PubMed: 14688492]

PILL-collaborative 2011 . PILL-collaborative. Rodgers A, Patel A, Berwanger O, Bots M, Grimm R, et al. An international randomised placebo-controlled trial of a four-component combination pill (“polypill”) in people with raised cardiovascular risk. Plos One. 2011; 6(5):e19857. [PubMed: 21647425]

Preston 2007 . Preston RA, Harvey P, Herfert O, Dykstra G, Jukema JW, Sun F, et al. A randomized, placebo-controlled trial to evaluate the efficacy, safety, and pharmacodynamic interaction of coadministered amlodipine and atorvastatin in 1660 patients with concomitant hypertension and dyslipidemia: the respond trial. J Clin Pharmacol. 2007; 47(12):1555-69. [PubMed: 18048574]

Rashid 2003 . Rashid P, Leonardi-Bee J, Bath P. Blood pressure reduction and secondary prevention of stroke and other vascular events: a systematic review. Stroke; a journal of cerebral circulation. 2003; 34(11):2741-8.

Sever 2003 . Sever PS, Dahlof B, Poulter NR, Wedel H, Beevers G, Caulfield M, et al. Prevention of coronary and stroke events with atorvastatin in hypertensive patients who have average or lowerthan-average cholesterol concentrations, in the Anglo-Scandinavian Cardiac Outcomes Trial-Lipid Lowering Arm (ASCOT-LLA): a multicentre randomised controlled trial. Lancet. 2003; 361(9364):1149-58. [PubMed: 12686036]

Steyn 2005 . Steyn K, Sliwa K, Hawken S, Commerford P, Onen C, Damasceno A, et al. Risk factors associated with myocardial infarction in Africa: the INTERHEART Africa study. Circulation. 2005; 112(23):3554-61. [PubMed: 16330696]

Taylor 2011 . Taylor F, Ward K, Moore TH, Burke M, Davey Smith G, Casas JP, et al. Statins for the primary prevention of cardiovascular disease. Cochrane Database of Systematic Reviews. 2011; (Issue 1) [DOI: 10.1002/14651858.CD004816.pub4].

Thomas 2002 . Thomas F, Bean K, Guize L, Quentzel S, Argyriadis P, Benetos A. Combined effects of systolic blood pressure and serum cholesterol on cardiovascular mortality in young. Eur Heart J. 2002; 23(7):528-35. [PubMed: 11922642]

TIPS 2009 . The Indian Polycap Study (TIPS). Effects of a polypill (Polycap) on risk factors in middle-aged individuals without cardiovascular disease (TIPS): a phase II, double-blind, randomised trial. Lancet. 2009; 373:1341-51. [PubMed: 19339045]

Turnbull 2003 . Turnbull F. Effects of different blood-pressure-lowering regimens on major cardiovascular events: results of prospectively-designed overviews of randomised trials. Lancet. 2003; 362(9395):1527-35. [PubMed: 14615107]

Vartiainen 2010 . Vartiainen E, Laatikainen T, Peltonen M, Juolevi A, Mannisto S, Sundvall J, et al. Thirty-five-year trends in cardiovascular risk factors in Finland. International journal of epidemiology. 2010; 39(2):504-18. [PubMed: 19959603] 
Viera 2011 . Viera AJ, Sheridan SL, Edwards T, Soliman EZ, Harris R, Furberg CD. Acceptance of a Polypill approach to prevent cardiovascular disease among a sample of U.S. physicians. Prev Med. 2011; 52(1):10-5. [PubMed: 20933538]

Wald 2003 . Wald NJ, Law MR. A strategy to reduce cardiovascular disease by more than $80 \%$. BMJ. 2003; 326(7404):1419. [PubMed: 12829553]

Wald 2011 . Wald DS, Wald NJ. The Polypill in the prevention of cardiovascular disease. Prev Med. 2011; 52(1):16-7. [PubMed: 21130112]

WHO 2002 . WHO. Secondary prevention of non-communicable disease in low and middle income countries through community-based and health service interventions. Report of WHO-Wellcome Trust meeting of experts; 1-3 August 2001; Geneva: World Health Organisation; 2002.

WHO 2004 . WHO. Burden of disease: DALYs. 2004. Available from:http://www.who.int/ healthinfo/global_burden_disease/GBD_report_2004update_part4.pdf

WHO 2005 . WHO. Preventing chronic disease: a vital investment. WHO global report. World Health Organisation; Geneva: 2005.

WHO 2008 . WHO. [Accessed on 02/01/2012] Global Health Observatory Data Respository. Available from http://apps.who.int/ghodata

WHO 2010 (a) . WHO. Global status report on noncommunicable diseases. World Health Organisation; Geneva: 2010 (a).

WHO 2010 (b) . WHO. Package of essential non-communicable (PEN) disease interventions for primary health care in low-resource settings. World Health Organisation; Geneva: 2010 (b).

Wong 2006 . Wong ND, Lopez V, Tang S, Williams GR. Prevalence, treatment, and control of combined hypertension and hypercholesterolemia in the United States. Am J Cardiol. 2006; 98(2):204-8. [PubMed: 16828593]

Yusuf 2002 . Yusuf S. Two decades of progress in preventing vascular disease. Lancet. 2002; 360(9326):2-3. [PubMed: 12114031]

Yusuf 2004 . Yusuf S, Hawken S, Ounpuu S, Dans T, Avezum A, Lanas F, et al. Effect of potentially modifiable risk factors associated with myocardial infarction in 52 countries (the INTERHEART study): case-control study. Lancet. 2004; 364(9438):937-52. [PubMed: 15364185]

Yusuf 2011 . Yusuf S, Islam S, Chow CK, Rangarajan S, Dagenais G, Diaz R, et al. Use of secondary prevention drugs for cardiovascular disease in the community in high-income, middleincome, and low-income countries (the PURE study): a prospective epidemiological survey. Lancet. 2011; 378(9798):1231-43. [PubMed: 21872920] * Indicates the major publication for the study 